\title{
Propagation pattern of low frequency waves in the terrestrial magnetosheath
}

\author{
Y. Narita and K.-H. Glassmeier \\ Institut für Geophysik und extraterrestrische Physik, Mendelssohnstr. 3, D-38106 Braunschweig, Germany \\ Received: 20 September 2006 - Accepted: 13 October 2006 - Published: 20 October 2006
}

\begin{abstract}
Propagation pattern (distribution of phase velocities) is determined in three dimensions in the terrestrial magnetosheath on a statistical basis using Cluster spacecraft observations. It is found that the anti-sunward propagation dominates and that the propagation direction is toward the magnetosheath flank at smaller zenith angles, while it is toward the magnetopause at larger angles. This pattern is axially symmetric regardless of the interplanetary magnetic field direction and agrees qualitatively with the density gradient directions in a hydromagnetic flow model of the magnetosheath, suggesting that the wave refraction mechanism is more significant than the wave drift effect.
\end{abstract}

Keywords. Magnetospheric physics (Magnetosheath; Plasma waves and instabilities) - Space plasma physics (Waves and instabilities)

\section{Introduction}

Identifying a wave propagation pattern is not an easy problem in the magnetosheath, since the ambient magnetic field is bent to the magnetopause shape, which causes an asymmetry about the zenith axis (GSM-X direction). In the case of the foreshock or the solar wind, in contrast, the ambient field can be well approximated by a homogeneous field and the wave propagation pattern can be relatively easily configured and sketched (Narita et al., 2004). The bent magnetic field may cause an asymmetry in the wave propagation pattern in the magnetosheath, for instance, between the plane containing the bent field and the one perpendicular to it, but it is not clear how similar or how different the propagation pattern is around the symmetry axis of the magnetosheath. We use Cluster spacecraft data for a statistical study and determine distributions of wave propagation directions. Fur-

Correspondence to: Y. Narita

(y.narita@ tu-bs.de) thermore, the use of the four identical spacecraft enables us to determine intrinsic phase speeds in the plasma rest frame (hereafter, plasma frame).

Although a number studies have been presented on the magnetosheath waves, most of them focused on the wave mode identification, e.g. dispersion relations (Narita et al., 2005), transport ratios (Denton et al., 1998), spectral signatures (Anderson et al., 1994), and the propagation pattern is yet poorly understood. Furthermore, earlier studies of the propagation directions relied much on Alfvénic fluctuations in the magnetosheath. Matsuoka et al. (2000) investigated the Alfvénic fluctuations observed by Geotail spacecraft and found that the propagation direction is outward from the symmetry axis, which was directly confirmed by Cluster spacecraft later on (Schäfer et al., 2005). On the other hand, signs of the mirror mode are more often observed in the magnetosheath (Tsurutani et al., 1982; Lucek et al., 1999). Recently it was found that the mirror mode waves propagate toward the magnetopause in the plane containing the interplanetary magnetic field direction (Narita et al., 2006). We aim to identify the propagation pattern in the magnetosheath in "three dimensions" in the present paper.

\section{Statistical study}

The purpose of study is to determine spatial distributions of the phase velocity vectors at various coordinates in the magnetosheath on a statistical basis. For this purpose the Cluster spacecraft mission (Escoubet et al., 2001) offers a unique possibility. It consists of four identical spacecraft and therefore enables to determine the wave vectors and the phase velocity vectors. The time intervals of the Cluster observations in the magnetosheath are chosen for the mission phase with the smallest spacecraft separation $(100 \mathrm{~km})$ from February to June 2002, from which 84 magnetosheath traversals are investigated. 


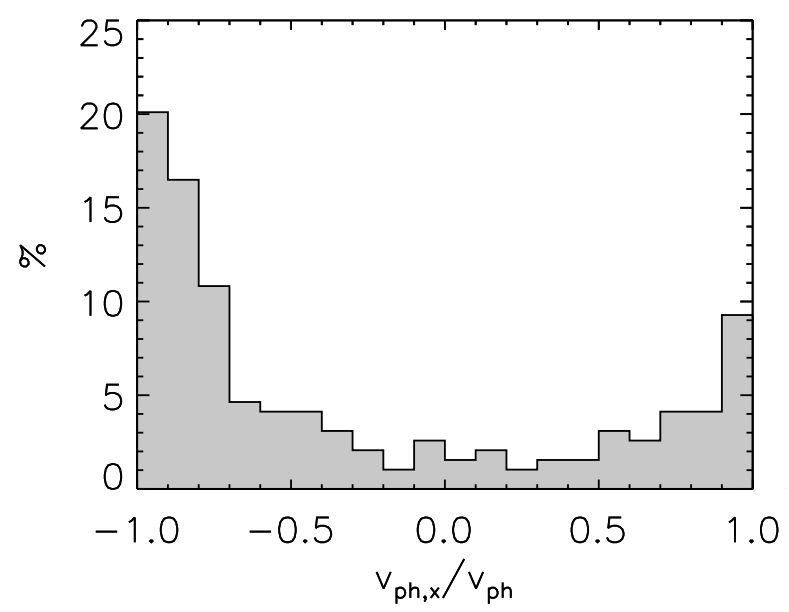

Fig. 1. Histogram of sunward component of phase velocities (in the plasma frame) divided by the magnitude of phase velocities.

It is convenient to introduce the solar wind coordinate system (Narita et al., 2006; Verigin et al., 2006). Its basis vectors are spanned as

$\boldsymbol{e}_{\boldsymbol{x}}=-\boldsymbol{e}_{\boldsymbol{v}}$

$\boldsymbol{e}_{\boldsymbol{y}}=\boldsymbol{e}_{z} \times \boldsymbol{e}_{\boldsymbol{x}}$

$\boldsymbol{e}_{z}=-\frac{B_{x}}{\left|B_{x}\right|} \boldsymbol{e}_{\boldsymbol{v}} \times \boldsymbol{e}_{\boldsymbol{b}}$

where $\boldsymbol{e}_{\boldsymbol{v}}$ and $\boldsymbol{e}_{\boldsymbol{b}}$ denote the unit vectors in the solar wind direction and in the interplanetary magnetic field (IMF) direction, respectively. $B_{x}$ denotes the sunward component of the IMF. In the solar wind coordinate system the IMF is tangent to the bow shock in the $Y>0$ region. Therefore the quasiparallel and the quasi-perpendicular shock regimes are separated into the $Y<0$ and $Y>0$ areas, respectively. The IMF is contained in the XY plane. The Cluster observations are limited to the high latitude regions in the magnetosheath because of its polar orbit with apogee about $20 R_{E}\left(1 R_{E}=6400 \mathrm{~km}\right)$, but they can widely spread out from the low to the high latitudes in the solar wind coordinate system, as the XY plane is rotated about the $X$ axis to include the IMF direction. The ACE spacecraft data of the ions and the magnetic field are used to determine $\boldsymbol{e}_{\boldsymbol{v}}, \boldsymbol{e}_{\boldsymbol{b}}$, and $B_{x}$ for the respective Cluster observations. The IMF as well as the bent magnetic field pattern in the magnetosheath are conveniently represented in the XY plane in this coordinate system.

In the wave analysis procedure dominant frequencies are first identified in the frequency spectra using Cluster magnetic field data (Balogh et al., 2001), and then wave vectors at those frequencies are identified. We make use of the wave telescope method (Glassmeier et al., 2001; Motschmann et al., 1996; Pinçon and Lefeuvre, 1991) which was developed particularly for the multi-spacecraft mission. The Doppler shift of the frequencies is then corrected and the

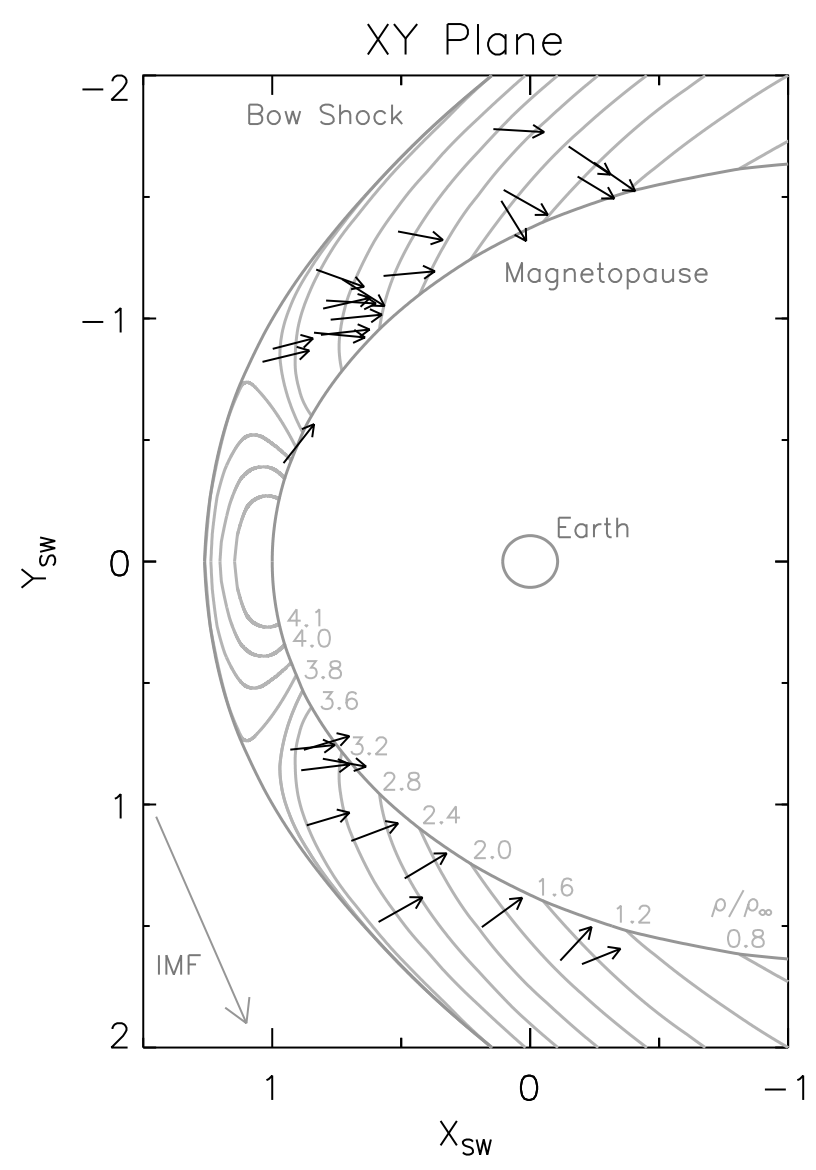

Fig. 2. Distribution of the phase velocity vectors in the XY plane of the solar wind coordinate system. Contours represent density ratio to the upstream one in the Spreiter model.

plasma frame frequencies are determined using the relation $\omega_{p l}=\omega_{s c}-\boldsymbol{k} \cdot \boldsymbol{v}_{\boldsymbol{f}}$, where $\omega_{p l}$ denotes the plasma frame frequency, $\omega_{s c}$ the spacecraft frame frequency, $\boldsymbol{k}$ the wave vector, and $\boldsymbol{v}_{f l}$ the plasma flow velocity. Cluster ion data are used to determine $\boldsymbol{v}_{\boldsymbol{f l}}$ (Rème et al., 2001). It should be noted that only multi-point measurements can determine the plasma frame frequencies, since one needs the wave vector $\boldsymbol{k}$ when applying the Doppler relation. Finally the phase velocity vectors are determined from $\omega_{p l}$ and $\boldsymbol{k}$ as $\boldsymbol{v}_{\boldsymbol{p} \boldsymbol{h}}=\omega_{p l} \boldsymbol{k} / \boldsymbol{k}^{2}$ at the observation coordinates $\boldsymbol{r}$ which are scaled from the magnetopause to the bow shock crossing position.

\section{Results}

Both sunward and anti-sunward propagation (in the plasma frame) are identified in the magnetosheath, but the antisunward propagation dominates (Fig. 1). The majority of the waves peaks in this direction $\left(v_{p h, x} / v_{p h} \sim-1\right)$ and hence the anti-sunward direction is the most preferred direction. 


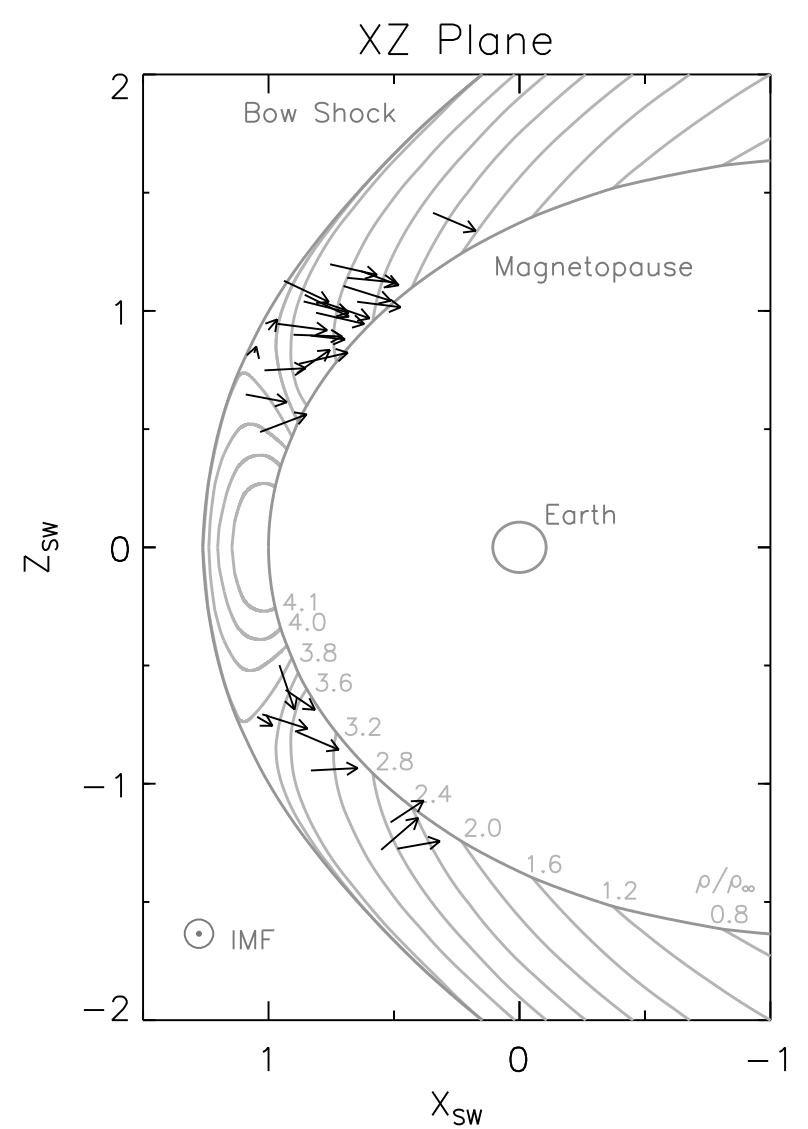

Fig. 3. The same panels as Fig. 2 for the $\mathrm{XZ}$ plane.

The propagation pattern for the anti-sunward waves exhibits a unique organization shown in detail below. Figure 2 displays the distribution of the phase velocities in the XY plane (containing the IMF direction) in the solar wind coordinate system. They are plotted as unit vectors projected to this plane. The observation coordinates in the magnetosheath are scaled to the relative position from the magnetopause to the bow shock using the Spreiter model (Spreiter et al., 1966) of axially symmetric, hydromagnetic flow in the magnetosheath under the condition of Mach number $M=8$ and polytropic index $\gamma=5 / 3$. The anti-sunward propagation is clearly seen at the zenith angle (the angle from the $X$ axis) about $45^{\circ}$, while the propagation directions are slightly shifted toward the magnetopause at the zenith angle about $90^{\circ}$. This tendency is found both in the $Y>0$ and $Y<0$ half planes. Note that the Cluster spacecraft have an apogee typically upstream of the bow shock and the observations are not made in the subsolar region (near the $\mathrm{X}$ axis). Contours in gray in Fig. 2 represent the density ratio relative to the upstream value varying from 0.8 to 4.1 . It exhibits a maximum in the subsolar region and becomes diminished toward the flank region. Superposition of the phase velocity vectors and the density contours yields an interesting result, that is the phase velocities traverse more or less perpendicular to

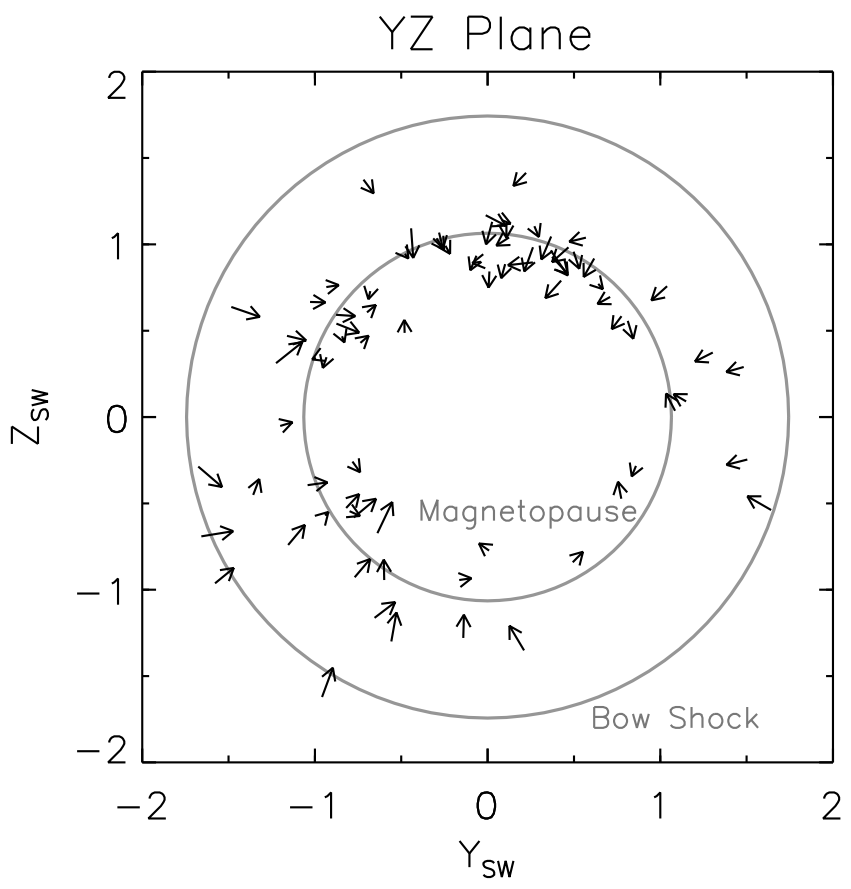

Fig. 4. Distribution of phase velocity vectors in the $\mathrm{YZ}$ plane.

the density contours toward the smaller densities, i.e. the relation $\boldsymbol{v}_{\boldsymbol{p} \boldsymbol{h}} \|-\nabla n$ holds, where $\boldsymbol{v}_{\boldsymbol{p} \boldsymbol{h}}$ and $\nabla n$ denote the phase velocity and the density gradient, respectively.

Figure 3 displays the distribution of the phase velocities in the XZ plane (perpendicular to the IMF). Again the above pattern is seen, too. The propagation is almost anti-sunward at the zenith angle about $45^{\circ}$. The waves at the smaller zenith angles tend to propagate in the flank direction, whereas those at the larger angles tend to propagate in the magnetopause direction.

Figure 4 displays the distribution in the $\mathrm{YZ}$ plane. Most of the waves propagate toward the $\mathrm{X}$ axis, $(Y, Z)=(0,0)$, at various clock angles in this plane. The length of the arrows are smaller, reflecting the fact that the phase velocities are dominant in the $\mathrm{X}$ direction.

\section{Conclusions}

To summarize, the anti-sunward propagation dominates. At the smaller zenith angles the propagation is toward the magnetosheath flank, and at the larger angles it is toward the magnetopause. At $45^{\circ}$ it is almost anti-sunward. The propagation directions form qualitatively a symmetric pattern around the $\mathrm{X}$ axis and it can be approximately expressed as $\boldsymbol{v}_{\boldsymbol{p} \boldsymbol{h}} \|-\nabla n$.

The relationship between the propagation pattern and the density distribution is of great interest. In plasmas waves may be coupled to the density gradient, and they become the so-called drift waves. In the case of the magnetosheath the mirror mode waves are often observed, and the existence of 
the drift mirror mode has been expected. The drift mirror mode, however, propagates perpendicular to the density gradient direction (Hasegawa, 1969; Pokhotelov et al., 2001), whereas our results prefer the parallel direction. Perhaps the drift mirror mode is operating in the magnetosheath, but the frequency is proportional to logarithm of the density distribution, $\omega \propto \nabla \log n$, and therefore the wave drift effect would be too small to detect. The parallel propagation to the density gradient may suggest a wave refraction in an inhomogeneous medium. In that case the WKB approximation or the ray tracing method should be applied to the low frequency waves in the magnetosheath, which solves the wave vector $\boldsymbol{k}$ as function of the coordinate $\boldsymbol{r}$. This shall be done and compared with the Cluster observations.

Acknowledgements. This work was financially supported by the Federal Ministry for Education and Research (Bundesministerium für Bildung und Forschung) and the German Aerospace Center (Deutsches Zentrum für Luft- und Raumfahrt) under contract 50OC0103. Y. Narita thanks H. Rème for providing the CIS-HIA data, D. J. McComas for the ACE SWEPAM data, and N. F. Ness for the ACE MAG data.

Topical Editor I. A. Daglis thanks B. Tsurutani for his help in evaluating this paper.

\section{References}

Anderson, B. J., Fuselier, S. A., Gary, S. P., and Denton, R. E.: Magnetic spectral signatures in the Earth's magnetosheath and plasma depletion layer, J. Geophys. Res., 99, 5877-5891, 1994.

Balogh, A., Carr, C. M., Acuña, M. H., Dunlop, M. W., Beek, T. J., Brown, P., Fornaçon, K.-H., Georgescu, E., Glassmeier, K.-H., Harris, J., Musmann, G., Oddy, T., and Schwingenschuh, K.: The Cluster magnetic field investigation: overview of in-flight performance and initial results, Ann. Geophys., 19, 1207-1217, 2001 ,

http://www.ann-geophys.net/19/1207/2001/.

Denton, R. E., Lessard, M. R., LaBelle, J. W., and Gary, S. P.: Identification of low-frequency magnetosheath waves, J. Geophys. Res., 103, 23 661-23 676, 1998.

Escoubet, C. P., Fehringer, M., and Goldstein, M.: The Cluster mission, Ann. Geophys., 19, 1197-1200, 2001,

http://www.ann-geophys.net/19/1197/2001/.

Glassmeier, K.-H., Motschmann, U., Dunlop, M., Balogh, A., Acuña, M. H., Carr, C., Musmann, G., Fornaçon, K.-H., Schweda, K., Vogt, J., Georgescu, E., and Buchert, S.: Cluster as a wave telescope - first results from the fluxgate magnetometer, Ann. Geophys., 19, 1439-1447, 2001 (Correction, Ann. Geophys., 21, 1071, 2003).

Hasegawa, A.: Drift mirror instability in the magnetosphere, Phys. Fluids, 12, 2642-2650, 1969.

Lucek, E. A., Dunlop, M. W., Balogh, A., Cargill, P., Baumjohann, W., Georgescu, E., Haerendel, G., and Fornaçon, K.-H.: Identification of magnetosheath mirror modes in Equator-S magnetic field data, Ann. Geophys., 17, 1560-1573, 1999, http://www.ann-geophys.net/17/1560/1999/.

Matsuoka, A., Southwood, D. J., Kokubun, S., and Mukai, T.: Propagation sense of low-frequency MHD waves in the magne- tosheath observed by Geotail, J. Geophys. Res., 105, $18361-$ $18376,2000$.

McComas, D. J., Bame, S. J., Barker, P., Feldman, W. C. Phillips, J. L., Riley, P., and Griffee, J. W.: Solar Wind Electron Proton Alpha Monitor (SWEPAM) for the Advanced Composition Explorer, Space Sci. Rev., 86, 563-612, 1998.

Motschmann, U., Woodward, T. I., Glassmeier, K.-H., Southwood, D. J., and Pinçon, J. L.: Wavelength and direction filtering by magnetic measurements at satellite arrays: generalized minimum variance analysis, J. Geophys. Res., 101, 4961-4965, 1996.

Narita, Y., Glassmeier, K.-H., Schäfer, S., Motschmann, U., Fränz, M., Dandouras, I., Fornaçon, K.-H., Georgescu, E., Korth, A., Rème, H., and Richter, I.: Alfvén waves in the foreshock propagating upstream in the plasma rest frame: statistics from Cluster observations, Ann. Geophys., 22, 2315-2323, 2004, http://www.ann-geophys.net/22/2315/2004/.

Narita, Y., and Glassmeier, K.-H.: Dispersion analysis of lowfrequency waves through the terrestrial bow shock, J. Geophys. Res., 110, A12215, doi:10.1029/2005JA011256, 2005.

Narita, Y., Glassmeier, K.-H., Fornaçon, K.-H. Richter, I., Schäfer, S., Motschmann, U., Dandouras, I., Rème, H., and Georgescu, E.: Low frequency wave characteristics in the upstream and downstream regime of the terrestrial bow shock, J. Geophys. Res., 111, A01203, doi:10.1029/2005JA011231, 2006.

Pinçon, J. L., and Lefeuvre, F.: Local characterization of homogeneous turbulence in a space plasma from simultaneous measurement of field components at several points in space, J. Geophys. Res., 96, 1789-1802, 1991.

Pokhotelov, O. A., Balikhin, M. A., Treumann, R. A., and Pavlenko, V. P.: Drift mirror instability revisited: 1 . Cold electron temperature limit, J. Geophys. Res., 106, 8455-8464, 2001.

Rème, H., Aoustin, C., Bosqued, J. M., Dandouras, I., et al.: First multispacecraft ion measurements in and near the Earth's magnetosphere with the identical Cluster ion spectrometry (CIS) experiment, Ann. Geophys., 19, 1303-1354, 2001, http://www.ann-geophys.net/19/1303/2001/.

Schäfer, S., Glassmeier, K.-H., Narita, Y. Fornaçon, K.-H., Fränz, M., and Dandouras, I.: Statistical phase propagation and dispersion analysis of low frequency waves in the magnetosheath, Ann. Geophys., 23, 3339-3349, 2005,

http://www.ann-geophys.net/23/3339/2005/.

Smith, C. W., L'Heureux, J., Ness, N. F., Acuña, M. H., Burlaga, L. F., and Scheifele, J.: The ACE Magnetic Fields Experiment, Space Sci. Rev., 86, 611-632, 1998.

Spreiter, J. R., Summers, A. L., and Alksne, A. Y.: Hydromagnetic flow around the magnetosphere, Planet. Space Sci., 14, 223-253, 1966.

Stone, E. C., Frandsen, A. M., Mewaldt, R. A., Christian, E. R., Margolies, D., Ormes, J. F., and Snow, F.: The Advanced Composition Explorer, Space Sci. Rev., 86, 1-22, 1998.

Tsurutani, B. T., Smith, E. J., Anderson, R. R., Ogilvie, K. W., Scudder, J. D., Baker, D. N., and Bame, S. J.: Lion roars and nonoscillatory drift mirror waves in the magnetosheath, J. Geophys. Res., 87, 6060-6072, 1982.

Verigin, M. I., Tátrallyay, M., Erdős, G., and Kotova, G. A.: Magnetosheath - interplanetary medium reference frame: application for a statistical study of mirror type waves in the terrestrial plasma environment, Adv. Space Res., 37, 515-521, 2006. 